\title{
Pharmacogenetics and nutritional supplementation in age-related macular degeneration
}

This article was published in the following Dove Press journal:

Clinical Ophthalmology

15 May 2015

Number of times this article has been viewed

\author{
Blake M Hampton \\ Jaclyn L Kovach \\ Stephen G Schwartz \\ Department of Ophthalmology, \\ Bascom Palmer Eye Institute, \\ University of Miami Miller School \\ of Medicine, Miami, FL, USA
}

Correspondence: Stephen G Schwartz Bascom Palmer Eye Institute, 3II 9th Street North, 100,

Naples, FL 34I02, USA

$\mathrm{Tel}+\mathrm{I} 2396593937$

Fax +I 2396593982

Email sschwartz2@med.miami.edu

\begin{abstract}
The Age-Related Eye Disease Study (AREDS) recommended treatment with antioxidants plus zinc in patients with intermediate or advanced age-related macular degeneration in order to reduce progression risks. Recent pharmacogenetic studies have reported differences in treatment outcomes with respect to variants in genes for $C F H$ and $A R M S 2$, although the treatment recommendations based on these differences are controversial. Different retrospective analyses of subsets of patients from the same AREDS trial have drawn different conclusions. The practicing clinician, who is not an expert on genetics, clinical trial design, or statistical analysis, may be uncertain how to interpret these results. Based on the balance of the available literature, we suggest not changing established practice recommendations until additional evidence from clinical trials becomes available.
\end{abstract}

Keywords: Age-Related Eye Disease Study (AREDS), age-related macular degeneration, agerelated maculopathy susceptibility 2 ( $A R M S 2)$, complement factor $\mathrm{H}(C F H)$, pharmacogenetics, randomized clinical trial (RCT)

\section{Introduction}

Age-related macular degeneration (AMD) remains the leading cause of irreversible visual loss among the elderly in developed nations. ${ }^{1}$ In 2004 , the prevalence of AMD in the US was estimated to be $1.47 \%$, indicating approximately 1.75 million patients, and was projected to increase to 2.95 million by $2020 .^{2}$

The Age-Related Eye Disease Study (AREDS) was a double-masked randomized clinical trial (RCT) evaluating nutritional supplementation on the progression of AMD. Study participants were graded by color fundus photography by a reading center. AMD category 1 patients had fewer than five small $(<63 \mu \mathrm{m})$ drusen; category 2 patients had multiple small drusen, non-extensive intermediate (63-124 $\mu \mathrm{m})$ drusen, and/or pigment abnormalities; category 3 patients had at least one large $(>125 \mu \mathrm{m})$ druse, extensive intermediate drusen, and/or non-central geographic atrophy; and category 4 patients had central geographic atrophy, neovascular AMD, or visual loss resulting from AMD in one eye. These patients were randomized into four treatment groups: antioxidants (including beta-carotene, vitamin $\mathrm{C}$ and vitamin $\mathrm{E}$ ), zinc (including zinc plus copper), antioxidants plus zinc, and placebo. Of enrolled patients, $96 \%$ were white. Treatment with antioxidants plus zinc was associated with a statistically significant reduction in disease progression in patients with intermediate (category 3 ) or advanced (category 4) AMD by about $25 \%$ after 5 years. ${ }^{3}$

A subsequent RCT, AREDS2, reported no further benefit with the addition of lutein plus zeaxanthin, docosahexaenoic acid plus eicosapentaenoic acid, or both. ${ }^{4}$ 
Because of the reported risks of lung cancer in ex-smokers associated with beta-carotene, ${ }^{5}$ many clinicians choose a formula containing lutein plus zeaxanthin rather than beta-carotene (in the original AREDS formula) in clinical practice.

Even with AREDS nutritional therapy, there appears to be substantial inter-patient variability in disease progression rates, which suggests a potential pharmacogenetic component to treatment response. The pharmacogenetics of various AMD treatments represent a subject of intense study. Numerous small case series have reported significant associations between treatment outcomes with photodynamic therapy and anti-VEGF therapy of neovascular AMD with variants in the genes for $C F H, A R M S 2, H T R A 1$, and others. ${ }^{6}$ However, two large RCTs, the Comparison of AMD Treatments Trials $(\mathrm{CATT})^{7}$ and the Inhibit VEGF in Patients with Age-Related Choroidal Neovascularization (IVAN) Study, ${ }^{8}$ reported no statistically significant associations.

Interestingly, there may be a stronger pharmacogenetic association with AREDS nutritional therapy than with photodynamic therapy or anti-VEGF treatments. Recent literature has focused on two genes (Table 1). In $\mathrm{CFH} \mathrm{Y402H,}$ thymine $(\mathrm{T})$ is substituted for cytosine $(\mathrm{C})$, the risk allele, resulting in an amino acid substitution from tyrosine (Y) to histidine (H). In ARMS2 A69S, guanine (G) is substituted for $\mathrm{T}$, the risk allele, resulting in an amino acid substitution from alanine (A) to serine $(\mathrm{S})$.

\section{Literature review}

Following publication of the original AREDS report, ${ }^{3}$ Klein et al retrospectively studied a subset of 876 white patients from the trial with categories 3 and 4 (ie, intermediate or advanced) AMD. They reported that, using multivariate analysis, after controlling for age, sex, education, smoking, and body mass index (BMI), the CFH TT variant was associated with a significantly more favorable treatment response than was the $C F H \mathrm{CC}$ variant in patients treated with both antioxidants and zinc. ${ }^{9}$ These results were intriguing but the investigators did not recommend further action, because no alternative treatment was suggested.

Recently, Awh et al retrospectively studied a subgroup of 995 white patients from the same AREDS trial with intermediate (category 3) AMD in at least one eye. They reported that, using multivariate analysis, after controlling for age, sex, education, smoking, and BMI, there were statistically significant differences in outcomes based on $C F H$ and $A R M S 2$ genotypes. The investigators recommended treatment of patients with no $C F H$ risk alleles (ie, $C F H$ TT) and with one or two $A R M S 2$ risk alleles (ie, ARMS2 GT or ARMS2 TT) with zinc only, and they recommended treatment of patients with one or two $C F H$ risk alleles (ie, $C F H \mathrm{CT}$ or $C F H \mathrm{CC}$ ) and no $A R M S 2$ risk alleles (ie, $A R M S 2 \mathrm{GG}$ ) with antioxidants only. ${ }^{10}$

Following this publication, the AREDS investigators reported an "unplanned retrospective evaluation" of 1,237 white study participants in the same AREDS trial. They concluded that variants at $C F H$ and $A R M S 2$ did not significantly affect the benefits of the AREDS nutritional supplements (antioxidants plus zinc). ${ }^{11}$

Awh et al subsequently analyzed 989 white study participants from the same AREDS trial and described four combinations of risk variants, based on alleles at $\mathrm{CFH}$ and $A R M S 2$. They reported that in patients with zero or one $C F H$ risk alleles (ie, $C F H$ TT or $C F H \mathrm{CT}$ ) and no $A R M S 2$ risk alleles (ie, ARMS2 GG), treatment with antioxidants alone was associated with best outcomes. In patients with two $C F H$ risk alleles (ie, $C F H C C$ ) and no $A R M S 2$ risk alleles (ie, $A R M S 2 \mathrm{GG}$ ), treatment with zinc or antioxidants plus zinc (the original AREDS formulation) was associated with worse outcomes than with placebo. In patients with zero or one $C F H$ risk alleles (ie, $C F H$ TT or $C F H$ CT) and one or two ARMS2 risk alleles (ie, ARMS2 GT or ARMS2 TT), treatment with antioxidants plus zinc or zinc was associated with better visual outcomes than with placebo. In patients with two $C F H$ risk alleles (ie, $C F H \mathrm{CC}$ ) and one or two ARMS2 risk alleles (ie, $A R M S 2 \mathrm{GT}$ or ARMS2 TT), no treatment was better than placebo. ${ }^{12}$ The authors recommended using these results to offer genotypedirected nutritional supplementation to patients.

Recently, Chew et al analyzed a subgroup of 526 patients from the original AREDS trial that was not previously analyzed by Awh et al (the "residual cohort") in an attempt to replicate their findings. They reported that, in the "residual cohort", the combination of antioxidants plus zinc was beneficial in all genetic subtypes described by Awh et al. ${ }^{13}$

\section{Discussion}

AMD is a complex genetic disease rather than a monogenic (Mendelian) disorder. The presence of one or more risk alleles does not necessarily result in a more affected phenotype, and

Table I Selected gene variants for age-related macular degeneration

\begin{tabular}{llll}
\hline Gene variant & Nucleotide substitution & Risk allele & Amino acid substitution \\
\hline CFH Y402H & T to C (thymine to cytosine) & C (cytosine) & Y to H (tyrosine to histidine) \\
ARMS2 A69S & G to T (guanine to thymine) & (thymine) & A to S (alanine to serine) \\
\hline
\end{tabular}


the absence of risk alleles does not necessarily result in a less affected phenotype. Therefore, genetic testing in AMD may give misleading results, exposing patients to increased risks based on potentially faulty assumptions about the predictive value of the testing. ${ }^{14}$

When reviewing these genetic studies, several potential sources of bias should be considered. Phenotypes were determined by color fundus photographs at a reading center. Of note, AREDS category 4 included patients with central geographic atrophy, neovascular AMD, or both in the same eye. Since the publication of the original AREDS study, it has been reported that patients with simultaneous geographic atrophy and neovascular AMD may differ genetically from patients with either entity in isolation. ${ }^{15}$

Other potential sources of error, common to other genetic studies, may be considered. In many of these reports the subgroups analyzed are relatively small, frequently fewer than 100 participants per group, which represents another potential source of error. Hardy-Weinberg equilibrium calculations may be used to check for errors; the earlier reports by Awh et $\mathrm{l}^{10}$ and Chew et $\mathrm{al}^{11}$ both mention Hardy-Weinberg but the other three studies do not.

Taking all of this information into consideration, it appears difficult to reconcile the results of the five published pharmacogenetic studies, all of which retrospectively analyzed patients from the same AREDS trial. (Of note, none of these studies analyzed outcomes using the AREDS2 formulation). The implications are substantial, because the decision to use genetic information to guide nutritional supplementation in AMD patients implies a need to perform genetic testing on two to three million patients in the US alone. This would represent a major change in the routine clinical care of AMD.

How should practicing clinicians, who are not experts in clinical trial design, genetics, or statistical analysis, interpret these conflicting results? It is evident that a post hoc analysis of a subgroup of patients from a prospective RCT that was not designed to answer a specific question is not the same as a prospective RCT that was designed to answer the specific question. ${ }^{16} \mathrm{~A}$ second data set showing similar results (a replication group) might be very helpful, but one does not exist at this time. Chew et al have drawn opposite conclusions from Awh et al using the same original data set. Further, Chew et al specifically attempted to replicate the findings of Awh et al using the "residual cohort" and were unable to do so. ${ }^{13}$

Retrospective subgroup analysis may result in statistically significant associations that are not indicative of causal effects. The likelihood of inadvertent selection bias, resulting in statistically significant but clinically meaningless associations, increases with the number of subgroups.
Replicating the findings with an additional data set may help distinguish between relevant and spurious correlations. ${ }^{17} \mathrm{To}$ illustrate this principle, Chew et al reported that patients from the cohort analyzed by Awh et al with the astrological signs of Aries and Cancer demonstrated a harmful effect from zinc, but this statistically significant association was not confirmed in the "residual cohort". ${ }^{13}$

In 2012, a task force from the American Academy of Ophthalmology recommended avoidance of:

Routine genetic testing for genetically complex disorders like AMD [...] until specific treatment or surveillance strategies have been shown in 1 or more published clinical trials to be of benefit to individuals with specific diseaseassociated genotypes. ${ }^{18}$

Do the two studies by Awh et al satisfy this recommendation? In our opinion, the answer remains "not at this time", especially considering the current lack of a second (replication) data set. Large-scale genetic screening of AMD patients is costly, although it is currently economically feasible in at least some populations. Nevertheless, in our opinion the balance of available evidence does not support the use of genetic screening to guide routine clinical decisions in AMD patients at this time.

We believe that pharmacogenetics is an important area of research, especially with regards to nutritional supplementation for AMD patients, but that the balance of the available literature suggests that it would be preferable to continue offering AREDS or AREDS2 supplementation for all patients meeting clinical criteria without using genetic analysis. As we continue to collect additional RCT data, including ideally a second (replication) data set, the precise pharmacogenetic relationships may become more clear.

\section{Acknowledgments}

This study was partially supported by NIH Center Core Grant P30EY014801 and by an unrestricted grant from Research to Prevent Blindness, New York, NY.

\section{Disclosure}

Dr Schwartz has received consulting fees from Alimera and Bausch + Lomb, as well as speaking fees from ThromboGenics and writing fees from Vindico. The other authors have no conflicts of interest to disclose.

\section{References}

1. Resnikoff S, Pascolini D, Etya'ale D, et al. Global data on visual impairment in the year 2002. Bull World Health Organ. 2004;82(11):844-851.

2. Friedman DS, O'Colmain BJ, Munoz B, et al. Prevalence of age-related macular degeneration in the United States. Arch Ophthalmol. 2004; 122(4):564-572. 
3. Age-Related Eye Disease Study Research Group. A randomized, placebo-controlled, clinical trial of high-dose supplementation with vitamins $\mathrm{C}$ and $\mathrm{E}$, beta-carotene, and zinc for age-related macular degeneration and vision loss: AREDS report no. 8. Arch Ophthalmol. 2001;119(10):1417-1436.

4. Age-Related Eye Disease Study 2 Research Group. Lutein + zeaxanthin and omega-3 fatty acids for age-related macular degeneration: the Age-Related Eye Disease Study 2 (AREDS2) randomized clinical trial. JAMA. 2013;309(19):2005-2015.

5. Omenn GS, Goodman GE, Thornquist MD, et al. Effects of a combination of beta carotene and vitamin A on lung cancer and cardiovascular disease. N Engl J Med. 1996;334(18):1150-1155.

6. Schwartz SG, Brantley MA Jr. Pharmacogenetics and age-related macular degeneration. J Ophthalmol. 2011;2011:25249.

7. Hagstrom SA, Ying GS, Pauer GJ, et al. Pharmacogenetics for genes associated with age-related macular degeneration in the Comparison of AMD Treatments Trials (CATT). Ophthalmology. 2013;120(3): 593-599.

8. Lotery AJ, Gibson J, Cree AJ, et al. Pharmacogenetic associations with vascular endothelial growth factor inhibition in participants with neovascular age-related macular degeneration in the IVAN study. Ophthalmology. 2013;120(12):2637-2643.

9. Klein ML, Francis PJ, Rosner B, et al. CFH and LOC387715/ARMS2 genotypes and treatment with antioxidants and zinc for age-related macular degeneration. Ophthalmology. 2008;115(6):1019-1025.

10. Awh CC, Lane AM, Hawken S, Zanke B, Kim IK. CFH and ARMS2 genetic polymorphisms predict response to antioxidants and zinc in patients with age-related macular degeneration. Ophthalmology. 2013; 120(11):2317-2323.
11. Chew EY, Klein ML, Clemons TE, et al. No Clinically Significant Association between CFH and ARMS2 Genotypes and Response to Nutritional Supplements: AREDS Report Number 38. Ophthalmology. 2014;121(11):2173-2180.

12. Awh CC, Hawken S, Zanke BW. Treatment Response to Antioxidants and Zinc Based on CFH and ARMS2 Genetic Risk Allele Number in the Age-Related Eye Disease Study. Ophthalmology. 2015;122(1): $162-169$.

13. Chew EY, Klein ML, Clemons TE, Agron E, Abecasis GR. Genetic testing in persons with age-related macular degeneration and the use of the AREDS supplements: to test or not to test? Ophthalmology. 2015; 122(1):212-215.

14. Stone EM. Genetic testing for age-related macular degeneration: not indicated now. JAMA Ophthalmol. Epub 2015 Mar 19.

15. Grob S, Luo J, Hughes G, et al. Genetic analysis of simultaneous geographic atrophy and choroidal neovascularization. Eye (Lond). 2012; 26(8):1106-1113.

16. Schwartz SG. Re: Awh et al.: CFH and ARMS2 genetic polymorphisms predict response to antioxidants and zinc in patients with age-related macular degeneration (Ophthalmology 2013;120:2317-23). Ophthalmology. 2014;121(8):e38.

17. Wittes J, Musch DC. Should we test for genotype in deciding on Age-Related Eye Disease Study supplementation? Ophthalmology. 2015;122(1):3-5.

18. Stone EM, Aldave AJ, Drack AV, et al. Recommendations for genetic testing of inherited eye diseases: report of the American Academy of Ophthalmology task force on genetic testing. Ophthalmology. 2012;119(11): $2408-2410$.
Clinical Ophthalmology

\section{Publish your work in this journal}

Clinical Ophthalmology is an international, peer-reviewed journal covering all subspecialties within ophthalmology. Key topics include: Optometry; Visual science; Pharmacology and drug therapy in eye diseases; Basic Sciences; Primary and Secondary eye care; Patient Safety and Quality of Care Improvements. This journal is indexed on

\section{Dovepress}

PubMed Central and CAS, and is the official journal of The Society of Clinical Ophthalmology (SCO). The manuscript management system is completely online and includes a very quick and fair peer-review system, which is all easy to use. Visit http://www.dovepress.com/ testimonials.php to read real quotes from published authors. 